\title{
تأثير إستخدام خبث أفران مجموعة جياد الصناعية على جودة الخرسانة
}

\author{
علي حسين محمد علي*1، الطيب عبداللطيف أحمد حبيب1 \\ 1 قسم الهندسة المدنية، كلية الهندسة والعمارة، جامعة بحري، الخرطوم، السودان. \\ * Corresponding author: Ali Hussein (e-mail: alibass288@hotmail.com).
}

Article history: Received 11 March 2020, Received in revised form 4 November 2020, Accepted 11 November 2020

\begin{abstract}
The need to produce high performance concrete led the researchers to try to exploit the potentialities of natural or artificial materials so as to improve the properties and performance of concrete. Slag is an industrial disposal which considered as a secondary product of pig-iron, these disposal will cause a severe harm to the environment in case of not get rid of it. In this investigation iron furnace slag which is produced at Giad Group - Iron Factory has been used as an alternative of proportion on cement after being processed, since its main oxides are similar to those of cement. In the practical part of our research slag has been added to the concrete mix in two ways, first by using it to replace as cement by weight as a substitutions of cement in different percent [10, 20, 30]\% without changing in water-cement ratio $[\mathrm{W} / \mathrm{C}]$, second by using the slag with the same previous percentage in addition to $[15,25] \%$ to replace cement with reducing in water-cement ratio [W/C], and in addition superplasticizer [SP 901] has been added as percent from cement used in the mixture. The effect of slag was studied on the properties of fresh and hardened concrete including slump test and compressive strength in $[7,14,28]$ days were investigated. The results show improvement in all concrete specimens for the added percentage of the slag as replacement materials and the addition of superplasticizer in the production of concrete and improvement in its properties. However, there is a gradual reduction in slump measurements due to slag percent increase in case of the two methods.
\end{abstract}

مستخلص: إن الحاجة إلى إنتاج خرسانة ذات أداء متميز دفعت الباحثن إلى محاولة إستغلال مواد طبيعية او صناعية لتحسين خواص وأداء

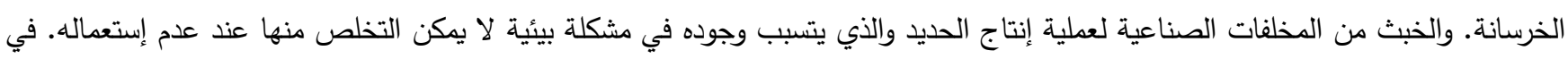
هذا البحث تم إستخدام خبث أفران صهر الحديد المنتج في مجموعة جياد الصناعية - مصنع الحديد من أفران القوس الكهربائي بطريقة التبريد

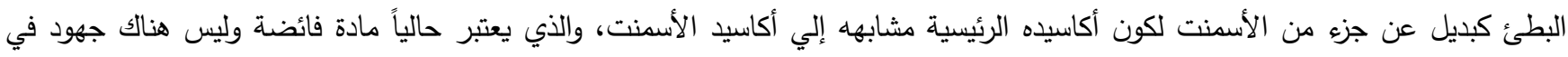

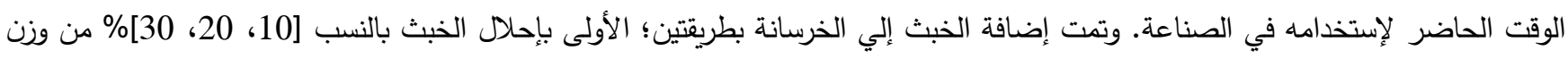

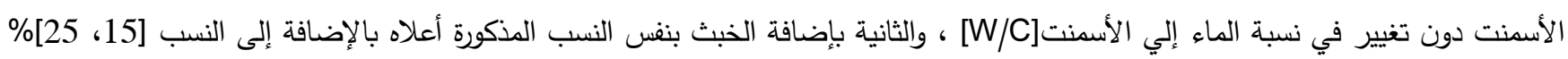

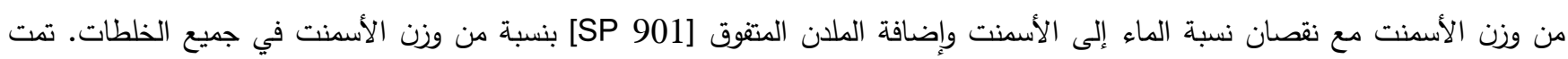

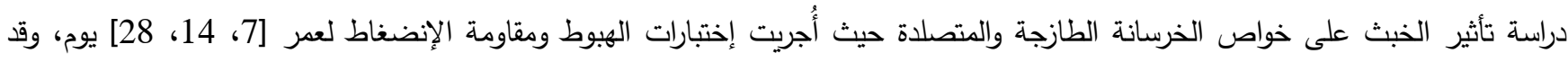

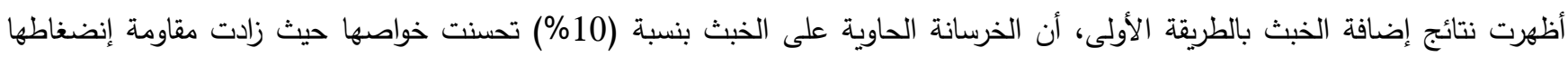
لعمر (28) يوم بنسبة (13\%) وبعدها تبدأ مقاومة الإنضغاط بالإنخفاض بزيادة نسبة الخبث بالمقارنة مع الخلطة المرجعية. وكذلك أظهائ أظهرت نتائج

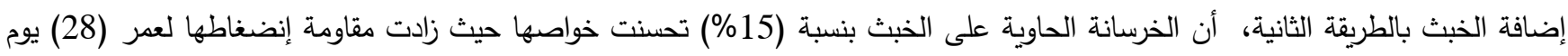

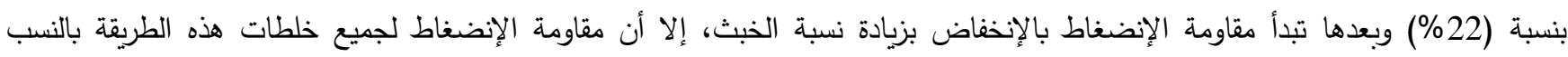

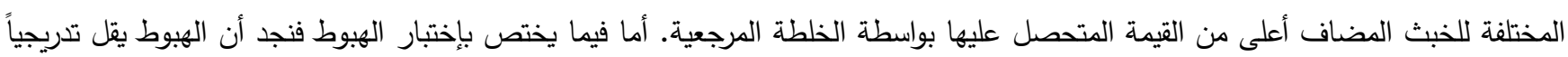
بزيادة نسبة الخبث المضاف للخلطة الخرسانية في كلِ من الطريقتين. الكلمات المفتاحية: الحديد، المخلفات الصناعية، الأسمنت، إحلال الخبث، الخرسانة، الخرسانة المتصلدة، قابلية التشغيل، مقاومة الإنضغاط، والخلطة المرجعية. 


$$
\text { يوجد نوعان من المواد الأولية هما: }
$$

(1) (أكاسيد الحديدالمسماة بتراب الحديد والتي هي الهيماتايت (Fe والمغناتايت

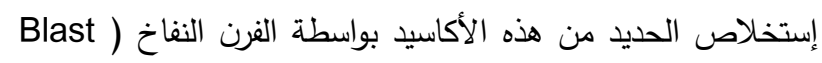
(Furnace مْ [2]، حيث يستخدم فحم الكوك المحتوي على كربون نسبته

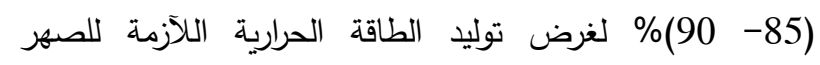
بالإضافة لتكوين أوكسيد الكربون (CO) ليقوم لبإختزال أكاسيد الحديد والممثلة وفقاً للمعادلات الكيميائية الآتية:

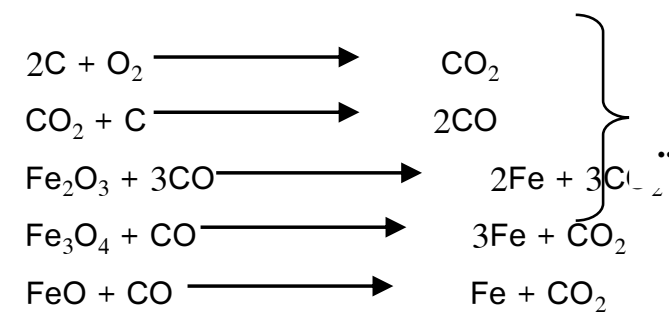

ولخفض درجة حرارة الإنصهار تستخدم كربونات الكالسيوم والمغنسيوم كمادة مصهرة (Flux Stone) والتي تتفكك حرارياً بدرجة حرارة (1000-900) مْ مكونة أوكسيد الكالسيوم (Lime) والذي يتحد ولند

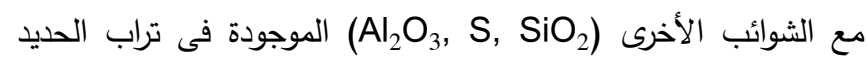

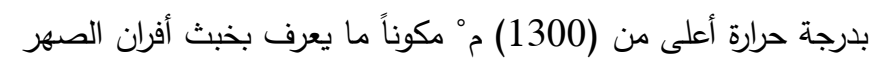

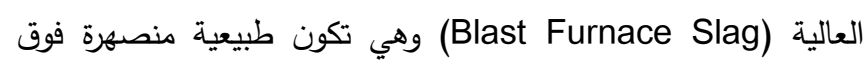
منصهر الحديد بسبب كثافتها القليلة بالمقارنة بكثافة الحديد وتتثِّكل

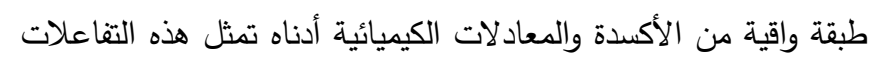

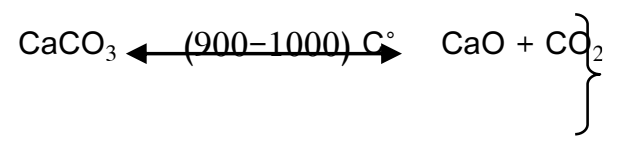

$\mathrm{MgCO}_{3} \longleftarrow(900-1000) \mathrm{C}: \mathrm{MgO}+\mathrm{CO}_{2}$

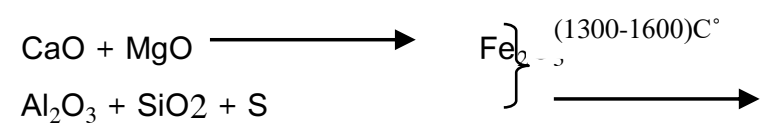

خبث أفران الصهر (Blast Furnace Slag)

(2) إستخدام الحديد الخردة (Scrap) كمادة أولية لإستخلاص الحديد والذي يستخلص بطرق عديدة منها:

i. الموقد المفتوح (Open Hearth).

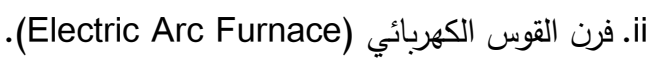

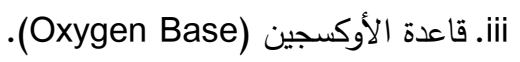

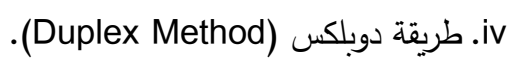

\section{1.تمهيد}

تعتبر الخرسانة من أكثر المواد الإنشائية التي شملتها البحوث

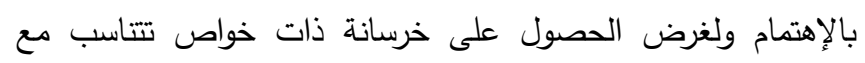
متطلبات المنشأ أو الظروف الجوية لابد من إستخدام المضافات. وفي خلاني

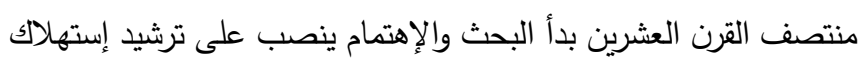

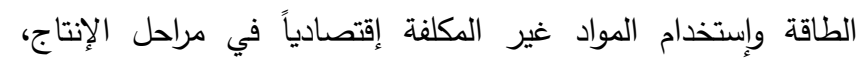

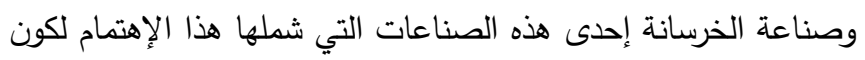
الأسمنت هو المادة الرئيسية فيها. أغلب الدراسات شملت ألتاعلت أمكانية تقليل

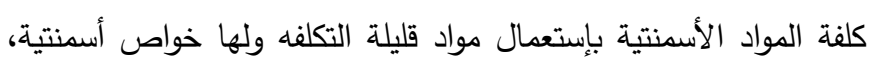
ونتيجة لذلك فقد تم إستعمال رقائق السليكا (Silica Fume) والمواد البوزولانية (Pozzolana) والخبث (Slag)، وقد أدى هذا إلى تقليل كلفة إنتاج الخرسانة وخاصة أن أغلب هذة المواد الجديدة هي نواتج

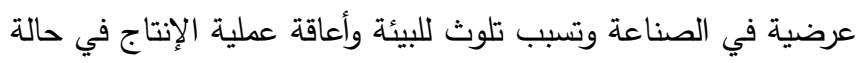

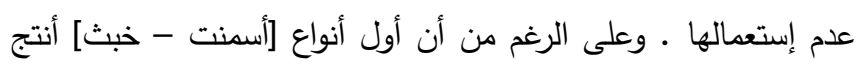

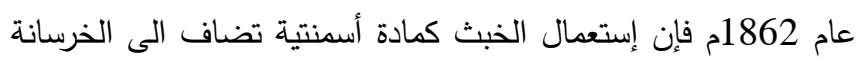
بشكل منفصل قد أعتمد في منتصف القرن الماضي وهو الثائع عالمياً

وينتج من أفران الصهر العالية [1]،[2].

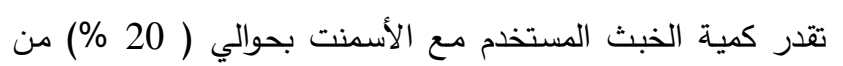
كمية الأسمنت المنتجة في أوربا [3]. وتختلف طرق إنتاجه ومعالجته الأن

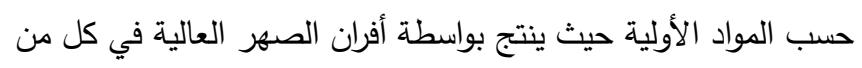
كندا وأستراليا ويسمى خبث أفران الصهر العالية (B.F.S)، أو ينتج (Furnace Electrical الخبث بواسطة الأفران القوسية الكهربائية

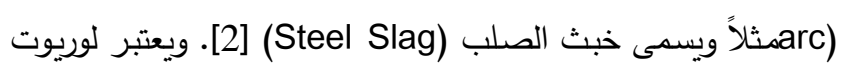
(Loriote) إنتاج مونه منه، وقد أخذت الدول المتقدمة المنتجة للخبث على المى محمل الجد بحث ودراسة وتطوير طرق معالجته حيث تم الإستفادة منه

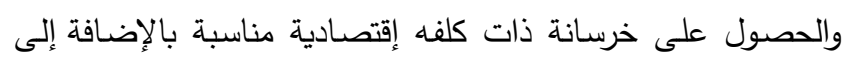

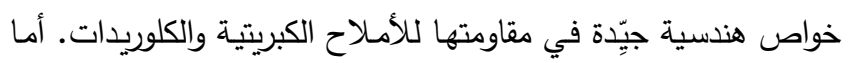

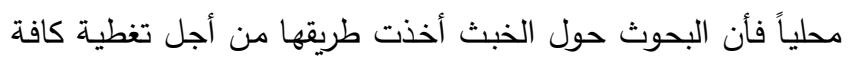
خواص الخبث المحلي وتحديد مدى الإستفادة منه.

\section{• هدف الدراسة}

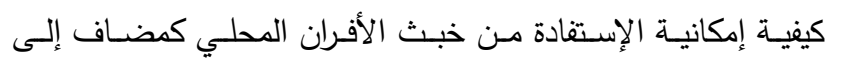

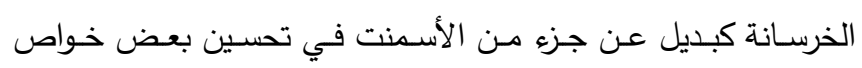

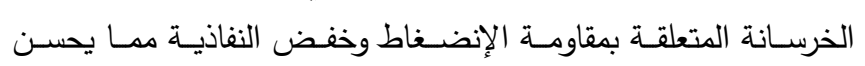
الخواص الأخرى لها.

\section{2. أنواع الخبث وطرق إنتاجه}

تختلف طرق إنتاج الخبث تبعًا إلى طرق إستخلاص الحديد والتي هي وطي إنتاجه

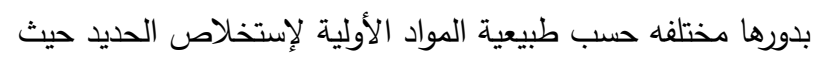




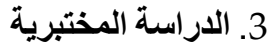

\section{• المواد الأولية المستخدمة}

تم إستخدام الأسمنت البورتلاندي الإعتيادي (O.P.C) السوداني

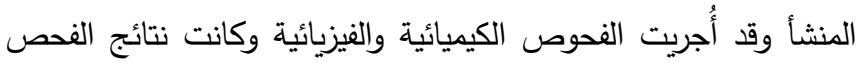
مطابقة للمواصفة القياسية السودانية (2011:170) [6]، وكما مُبيّن

بالجدولين (III) و (IV). - (IV).

وكما تم إستخدام الركام الناعم [الرمل] وتم فحصه ويوضِّح الجدول الفحوص الكيميائية والفيزيائية. وكذلك تم إستخدام الركام الخشن [الحصى] ذو مقاس أقصى قدره (20) مم وتم فحص الذواص الكيميائية والفيزيائية والجدول (VI) يوضِّح تلك الفحوص. ولخلط المكونات السابقة

الذكر تم إستخدام الماء الصالح للشرب لجميع الخلطات الخرسانية.

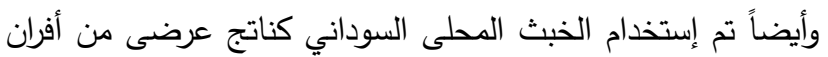
صهر المعادن الفولاذية من مجموعة جياد الصناعية - مصنع الحديد، حيث تم جلبه على شكل كتل بأحجام كبيره ومن ثم تكسيرها إلى أحجام أصغر وطحنها بإستخدام طاحونة ذات كرات فولاذية لغرض الحصول على تدرج مشابه إلى حد كبير لتدرج الأسمنت وبديلاً عنه فى الخلطات الخرسانية. وتم تحليل الخبث لمعرفة تركيبه الكيميائي بمعهد البحوث والإستشارات الصناعية [أنظر الجدول (I)]. وبالإضافة إلى ذلك تم إستخدام الملدن المتفوق (SP 901) سيرا بلاست لتقليل نسبة الماء فى الخلطة الخرسانية والذي يقلل من الفقدان الحاصل في قابلية التشغيل عند إضافة الخبث.

الجدول (III): الخو اص الكيميائية للأسمنت.

\begin{tabular}{|c|c|c|}
\hline حلود اللواصفةة القياسية & المحتوى (\%) & نوع الأوكسيا \\
\hline - & 61.94 & $\mathrm{CaO}$ \\
\hline- & 18.08 & $\mathrm{SiO}_{2}$ \\
\hline - & 5.58 & $\mathrm{Al}_{2} \mathrm{O}_{3}$ \\
\hline - & 2.43 & $\mathrm{Fe}_{2} \mathrm{O}_{3}$ \\
\hline الحد الأعلى (6\%) & 2.43 & $\mathrm{MgO}$ \\
\hline الحد الأعلى (3.5\%) & 2.93 & $\mathrm{SO}_{3}$ \\
\hline- & 0.99 & $\mathrm{~K}_{2} \mathrm{O}$ \\
\hline - & 0.18 & $\mathrm{Na}_{2} \mathrm{O}$ \\
\hline الحد الأعلى (3\%) & 4.4 & L.O.I \\
\hline
\end{tabular}

الجدول (IV): الخواص الفيزيائية للأسمنت.

\begin{tabular}{|c|c|c|}
\hline حدود المواصفة القياسية & النتائج & نوع الفحص \\
\hline الحد الأدنى & دقيقة 133 & فيكات - زمن الثُك الإبندائي (دقيقة) \\
\hline 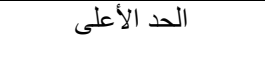 & دقيقة 205 & فيكات - زمن الثك النهائي (دقيقة) \\
\hline الحد الأدنى (22) & $\begin{array}{c}18.7 \\
32 \\
\end{array}$ & مقاومة الإنضغاط (نيوتن/ مع2) \\
\hline الحد الأعلى (0.8 \%) & 0.35 & 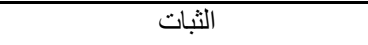 \\
\hline
\end{tabular}

إن الأسس الكيميائية لجميع الطرق أعلاه هي واحده وتتمثل فى أكسدة الثوائب الموجودة بالحديد الخردة [القديم] ويستخدم أوكسيد الهيد

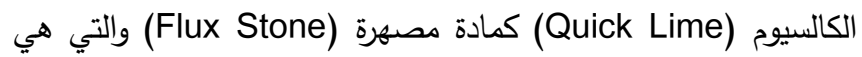

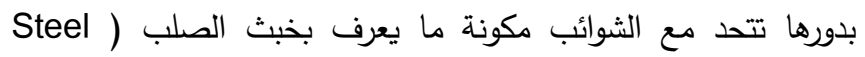
Slag) والذي يكون منصهر فوق منصهر الحديد [2]، والمعادلات الكيميائية الأتية تمثل هذه التفاعلات.

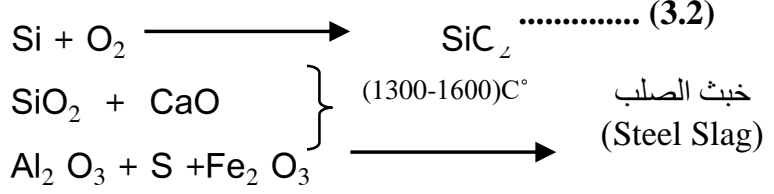

\section{التركيب الكيميائي للخبث:}

إن التركيب الكيميائي للخبث متغير بمدى كبير إعتماداً على طبيعه المواد الأولية مثل تراب الحديد والفحم، وعلى طريقة تصنيع الحديد حيث أن الأكاسيد الرئيسة المكونة للخبث هي (السيليكا، الألومنيا،

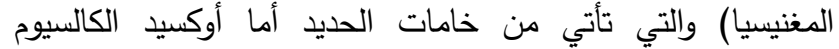
والمغنسيوم فصدرها كربونات الكالسيوم والمغنسيوم التي تضاف كمادة مساعدة مصهرة. أما الأكاسيد الثانوية فهي أوكسيد الحديد والكبريت

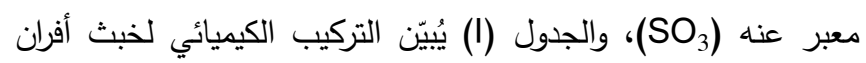
الصهر في بعض الدول ويلاحظ أن مجموع أكاسيد (السيليكا، الألومينا، الجير) تكوّنِ ما مجموعه نسبة (80\%) من المحتوى الكلي للأكاسيد

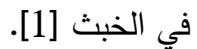

\begin{tabular}{|c|c|c|c|c|c|}
\hline $\begin{array}{c}\mathrm{Fe}_{2} \mathrm{O}_{3} \\
\%\end{array}$ & $\begin{array}{c}\mathrm{MgO} \\
\%\end{array}$ & $\begin{array}{c}\mathrm{Al}_{2} \mathrm{O}_{3} \\
\%\end{array}$ & $\begin{array}{r}\text { SiO } \\
\%\end{array}$ & $\begin{array}{c}\mathrm{CaO} \\
\%\end{array}$ & المصدر \\
\hline $0.7-0.3$ & $11-4$ & $\begin{array}{c}-12 \\
22\end{array}$ & $\begin{array}{c}-28 \\
36\end{array}$ & $\begin{array}{c}-36 \\
43\end{array}$ & بريطانيا \\
\hline $2.4-0.3$ & $18-0$ & $23-5$ & $\begin{array}{c}-34 \\
35\end{array}$ & $\begin{array}{c}-29 \\
48\end{array}$ & روسيا \\
\hline $3.8-0.5$ & $8-2$ & $\begin{array}{c}-13 \\
19\end{array}$ & $\begin{array}{c}-29 \\
36\end{array}$ & $\begin{array}{c}-40 \\
48\end{array}$ & فرنسا \\
\hline $1.0-0.2$ & $12-4$ & $\begin{array}{c}-10 \\
16\end{array}$ & $\begin{array}{c}-29 \\
35\end{array}$ & $\begin{array}{c}-38 \\
46\end{array}$ & ألمانيا \\
\hline $3.0-0.4$ & $21-7$ & $\begin{array}{c}-10 \\
22\end{array}$ & $\begin{array}{c}-28 \\
38\end{array}$ & $\begin{array}{c}-28 \\
39\end{array}$ & أفريقيا \\
\hline 22.31 & 1.71 & 18.82 & 32.51 & 23.06 & السوداد) \\
\hline
\end{tabular}

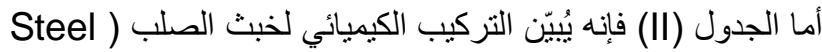

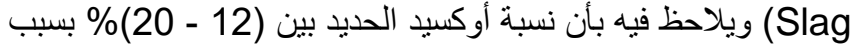

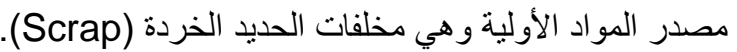
الجدول (II): التركيب الكيميائي لخبث الصلب.

\begin{tabular}{c|c|c|c|c|c}
\hline \hline $\mathrm{SO}_{3}$ & $\begin{array}{c}\mathrm{Fe}_{2} \mathrm{O}_{3} \\
\%\end{array}$ & $\begin{array}{c}\mathrm{MgO} \\
\%\end{array}$ & $\begin{array}{c}\mathrm{Al}_{2} \mathrm{O}_{3} \\
\%\end{array}$ & $\begin{array}{c}\mathrm{SiO} \\
\%\end{array}$ & $\begin{array}{c}\mathrm{CaO} \\
\%\end{array}$ \\
\hline $0.9-0.2$ & $20-12$ & $9-2$ & $4-3$ & $\begin{array}{c}-10 \\
20\end{array}$ & $\begin{array}{c}-40 \\
50\end{array}$ \\
\hline \hline
\end{tabular}


ولعمر (7، 14، 28) يوم، مع أخذ المتوسط الحسابي لثلاثة نماذج لكل

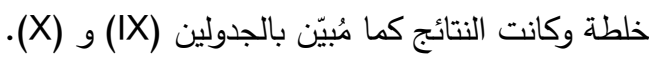
4. نتائج الدراسة والتحليل

الجدول (IX) والثكلان (1) و (2) تُوضِح نتائج فحصي الهيوط ومقاومة الإنضغاط للخرسانة المرجعية والخرسانة الحاوية على الخبث بالطريقة الأولى. وأيضاً الجدول (X) والثكلان (3) و (4) تُوضِّح نتائج فحصي الهبوط ومقاومة الإنضغاط للخرسانة المرجعية والخرسانة الحاوية على الخبث بالطريقة الثانية. أظهرت نتائج إضافة الخبث بالطريقة الأولى، أن الخرسانة الحاوية على الخبث بنسبة (10\%) أدت إلى تحسين خواص الخرسانة حيث زادت مقاومة إنضغاطها لعمر (28) يوم بنسبة (13\%) وبعدها تبدأ مقاومة الإنضغاط بالإنخفاض بزيادة نسبة الخبث بالمقارنة مع الخلطة المرجعية [Mix0] وذلك لأن الخبث المستخدم ذو نعومة عالية ويعمل

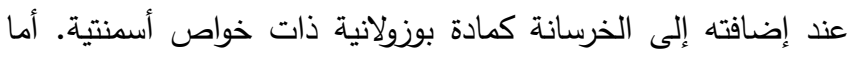

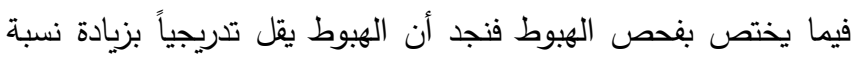
الخبث المضاف للخلطة الخرسانية [أنظر الثكل (2)]. الجدول (IX): نتائج إحلال الخبث بالطريقة الأولى.

\begin{tabular}{|c|c|c|c|c|c|}
\hline $\begin{array}{l}\text { Mix } \\
\text { No. }\end{array}$ & $\begin{array}{c}\text { Age } \\
\text { (Days) }\end{array}$ & $\begin{array}{c}\mathrm{W} / \mathrm{C}) \\
\%\end{array}$ & $\begin{array}{c}\text { Slag } \\
\%\end{array}$ & $\begin{array}{c}\text { Slump } \\
\text { (mm) }\end{array}$ & $\begin{array}{c}\text { Mean } \\
\mathrm{f}_{\mathrm{cu}} \\
(\mathrm{MPa})\end{array}$ \\
\hline Mix0 & \multirow{4}{*}{7} & \multirow{4}{*}{0.50} & - & 100 & 25.6 \\
\hline Mix1 & & & 10 & 80 & 29.25 \\
\hline Mix2 & & & 20 & 65 & 23.7 \\
\hline Mix3 & & & 30 & 53 & 15.8 \\
\hline Mix0 & \multirow{4}{*}{14} & \multirow{4}{*}{0.50} & - & 100 & 30.25 \\
\hline Mix1 & & & 10 & 80 & 31.8 \\
\hline Mix2 & & & 20 & 65 & 28.9 \\
\hline Mix3 & & & 30 & 53 & 17.8 \\
\hline & & & & & \\
\hline Mix0 & \multirow{4}{*}{28} & \multirow{4}{*}{0.50} & - & 100 & 32 \\
\hline Mix1 & & & 10 & 80 & 36.4 \\
\hline Mix2 & & & 20 & 65 & 31.17 \\
\hline Mix3 & & & 30 & 53 & 22.5 \\
\hline
\end{tabular}

الجدول (X): نتائج إحلال الخبث بالطريقة الثانية.

\begin{tabular}{|c|c|c|c|c|c|c|}
\hline $\begin{array}{l}\text { Mix } \\
\text { No. }\end{array}$ & $\begin{array}{c}\text { Age } \\
\text { (Days } \\
\text { ) }\end{array}$ & $\begin{array}{c}\mathrm{W} / \mathrm{C}) \\
\%\end{array}$ & $\begin{array}{c}\text { Slag } \\
\%\end{array}$ & $\begin{array}{c}\mathrm{SP} \\
\%\end{array}$ & $\begin{array}{c}\text { Slum } \\
\mathrm{p} \\
(\mathrm{mm})\end{array}$ & $\begin{array}{c}\text { Mean } \\
\mathrm{f}_{\mathrm{cu}} \\
(\mathrm{MPa} \\
)\end{array}$ \\
\hline Mix0 & \multirow{6}{*}{7} & 0.50 & - & - & 100 & 25.6 \\
\hline $\mathrm{Mix}^{*}{ }^{*}$ & & 0.45 & 10 & 1 & 85 & 33.5 \\
\hline Mix2* & & 0.40 & 15 & 1.2 & 73 & 33.6 \\
\hline Mix3* & & 0.40 & 20 & 1.2 & 68 & 33.1 \\
\hline Mix4* & & 0.38 & 25 & 1.5 & 60 & 32.7 \\
\hline Mix5* & & 0.38 & 30 & 1.5 & 45 & 31.8 \\
\hline
\end{tabular}

\begin{tabular}{|c|c|}
\hline النتائج & الخاصية \\
\hline 2.65 & الوزن النوعي \\
\hline$\% 0.6$ & الإمتصاص \\
\hline 1,692 (كجم/ م3) & الكثافة الكتلية \\
\hline$\% 0.28$ & $\mathrm{SO}_{3}$ \\
\hline
\end{tabular}

\begin{tabular}{|c|c|}
\hline النتائج & الخاصية \\
\hline 2.90 & الوزن النوعي \\
\hline$\% 0.8$ & الإمتصاص \\
\hline 1,610 (كجم/ م3) & الكثافة الكتلية \\
\hline$\% 0.04$ & $\mathrm{SO}_{3}$ \\
\hline
\end{tabular}

• الخلطات الخرسانية

الجدول (VII) يمثل الطريقة الأولى لإضافة الخبث بنسب مختلفة من

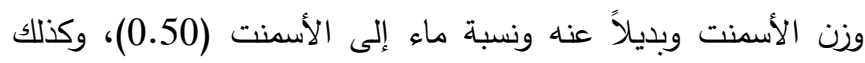
المكونات الأساسية للخلطة المرجعية. الجدول (VII): الدكونات الأساسية لخلطات الطريقة الأولى.

\begin{tabular}{|c|c|c|c|c|c|}
\hline $\begin{array}{l}\text { Mix } \\
\text { No. }\end{array}$ & $\begin{array}{c}\text { Cement } \\
\mathrm{Kg} / \mathrm{m}^{3}\end{array}$ & $\begin{array}{l}\text { Gravel } \\
\mathrm{Kg} / \mathrm{m}^{3}\end{array}$ & $\begin{array}{l}\text { Sand } \\
\mathrm{Kg} / \mathrm{m}^{3}\end{array}$ & $\begin{array}{l}\text { Water } \\
\mathrm{Kg} / \mathrm{m}^{3}\end{array}$ & $\begin{array}{c}\text { Slag } \\
\%\end{array}$ \\
\hline Mix0" & 380 & 980 & 820 & 190 & - \\
\hline Mix1 & 342 & 980 & 820 & 190 & 10 \\
\hline Mix2 & 304 & 980 & 820 & 190 & 20 \\
\hline Mix3 & 266 & 980 & 820 & 190 & 30 \\
\hline
\end{tabular}
والجدول (VIII) يمثل الطريقة الثانية لإضافة الخبث بنسب مختلفة من وزن الأسمنت وبديلاً عنه ونسبة ماء إلى الأسمنت متغيرة وإضافة لإنة الملدن المتفوق (SP 901)، وكنلك المكونات الأساسية للخلطة

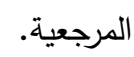

الجدول (VIII): المكونات الأساسية لخلطات الطريقة الثانية.

\begin{tabular}{c|c|c|c|c|c|c|c}
\hline \hline $\begin{array}{c}\text { Mix } \\
\text { No. }\end{array}$ & $\begin{array}{c}\text { Cement } \\
\mathrm{Kg} / \mathrm{m}^{3}\end{array}$ & $\begin{array}{c}\text { Gravel } \\
\mathrm{Kg} / \mathrm{m}^{3}\end{array}$ & $\begin{array}{c}\text { Sand } \\
\mathrm{Kg} / \mathrm{m}^{3}\end{array}$ & $\begin{array}{c}(\mathrm{W} / \mathrm{C}) \\
\%\end{array}$ & $\begin{array}{c}\text { Water } \\
\mathrm{Kg} / \mathrm{m}^{3}\end{array}$ & $\begin{array}{c}\text { Slag } \\
\%\end{array}$ & $\begin{array}{c}\mathrm{SP} \\
\%\end{array}$ \\
\hline Mix0 & 380 & 980 & 820 & 0.50 & 190 & - & - \\
\hline Mix1 $^{*}$ & 342 & 980 & 820 & 0.45 & 171 & 10 & 1 \\
\hline Mix2 $^{*}$ & 323 & 980 & 820 & 0.40 & 152 & 15 & 1.2 \\
\hline Mix3 $^{*}$ & 304 & 980 & 820 & 0.40 & 152 & 20 & 1.2 \\
\hline Mix4 $^{*}$ & 285 & 980 & 820 & 0.38 & 145 & 25 & 1.5 \\
\hline Mix5 $^{*}$ & 266 & 980 & 820 & 0.38 & 145 & 30 & 1.5 \\
\hline \hline
\end{tabular}

\section{• الفحوص المختبرية}

$$
\text { (1) فحص الهبوط }
$$

تم إجراء فحص الهبوط للخرسانة الطرية لجميع الخلطات الخرسانية التى تم إنتاجها في الدراسة. (2) فحص مقاومة الإنضغاط تم إجراء فحص مقاومة الإنضغاط بموجب المواصفة البريطانية (B.S 1881 - Part 4) 


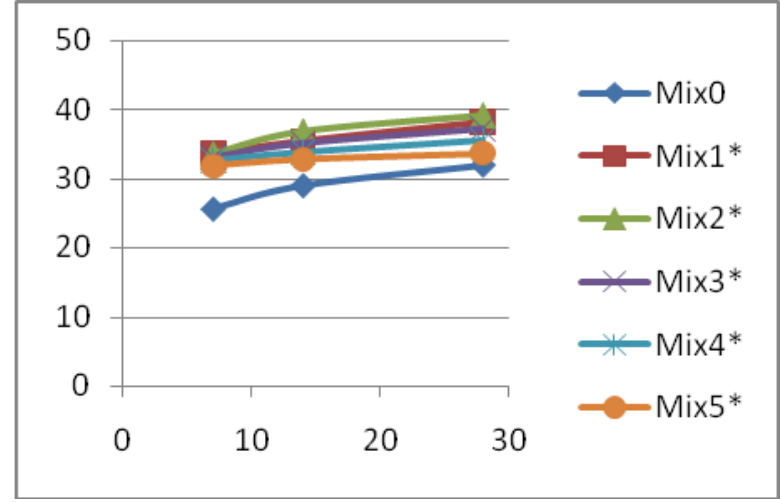

الثكل 3: نتائج فحص مقاومة الإنضغاط لخلطات الطريقة الثانية.

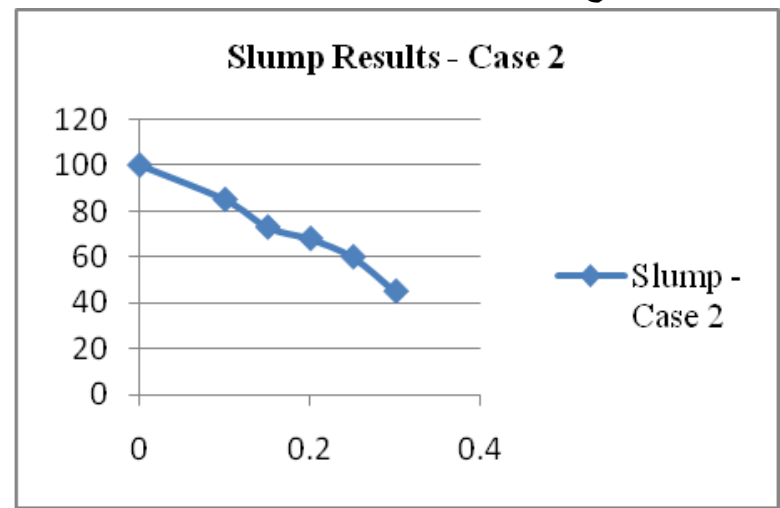

الثكل 4: نتائج فحص الهبوط لخلطات الطريقة الثانية.

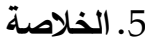

من خلال نتائج الفحوصات التي تم الحصول عليها ولجميع الخلطات الخرسانية المنتجة بالطريقتين يمكن إستخلاص الآتي:

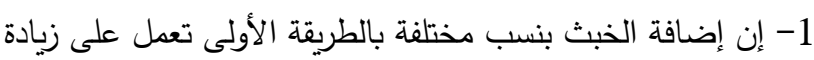
مقاومـة الإنضـغاط حتى نسبة (10\%) مـن وزن الأســنت [أنظر

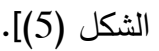

2- إن إضافة الخبث بنسب مختلفة بالطريقة الثانية تعمل على زيادة مقاومـة الإنضـغاط حتى نسبة (15\%) مـن وزن الأسـمنت [أنظـر

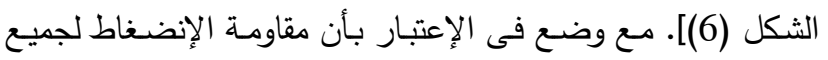
الخلطات الخرسانية المنتجة بهذه الطريقة أعلى من الخلطة المرجعية.

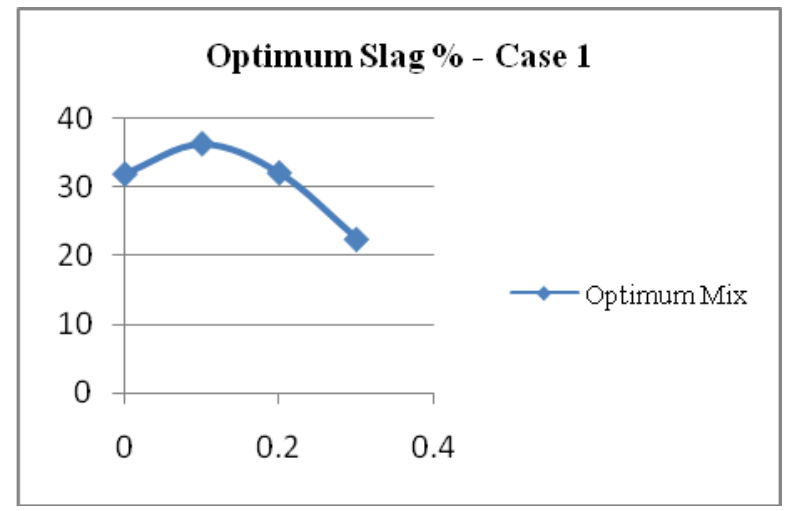

الثكل 5: نسبة إحلال الخبث المثلى (10\%) لخلطات الطريقة الأولى.

\begin{tabular}{|c|c|c|c|c|c|c|}
\hline 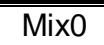 & \multirow{6}{*}{14} & $\overline{0.50}$ & - & $\overline{-1}$ & $\overline{100}$ & 29 \\
\hline $\operatorname{Mix} 1^{*}$ & & 0.45 & 10 & 1 & 85 & 35.4 \\
\hline Mix2 ${ }^{*}$ & & 0.40 & $\overline{15}$ & 1.2 & 73 & 36.8 \\
\hline Mix3* & & 0.40 & 20 & 1.2 & 68 & 35.1 \\
\hline Mix4* & & 0.38 & 25 & 1.5 & 60 & 33.9 \\
\hline Mix5* & & 0.38 & 30 & 1.5 & 45 & 32.8 \\
\hline Mix0 & \multirow{6}{*}{28} & 0.50 & - & - & 100 & 32 \\
\hline $\operatorname{Mix} 1^{*}$ & & 0.45 & 10 & 1 & 85 & 38.2 \\
\hline Mix2* & & 0.40 & 15 & 1.2 & 73 & 39.1 \\
\hline Mix3 ${ }^{*}$ & & 0.40 & 20 & 1.2 & 68 & 37.2 \\
\hline Mix4 $4^{*}$ & & 0.38 & 25 & 1.5 & 60 & 35.6 \\
\hline Mix5* & & 0.38 & 30 & 1.5 & 45 & 33.7 \\
\hline
\end{tabular}

وكذلك أظهرت نتائج إضافة الخبث بالطريقة الثانية، أن الخرسانة الحاوية على الخبث بنسبة (15\%) أدت إلى تحسين خواص الخرسانة حيث زادت مقاومة إنضغاطها لعمر (28) يوم بنسبة (22\%) وبعدها لإنها تبدأ مقاومة الإنضغاط بالإنخفاض بزيادة نسبة الخبث، إلا أن مقاومة

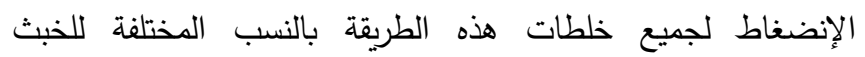
المضاف أعلى من القيمة المتحصل عليها بواسطة الخلطة المرجعية.

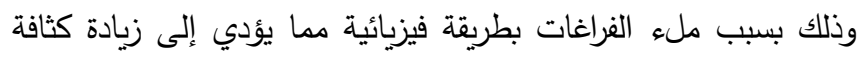

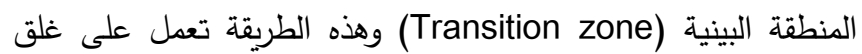
الفجوات الكبيرة التي كانت تشغلها جزيئات هيدروكسيد الكالسيوم

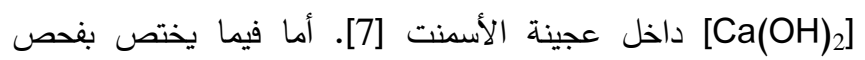

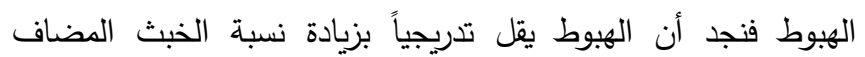
للخلطة الخرسانية [أنظر الشكل (4)].

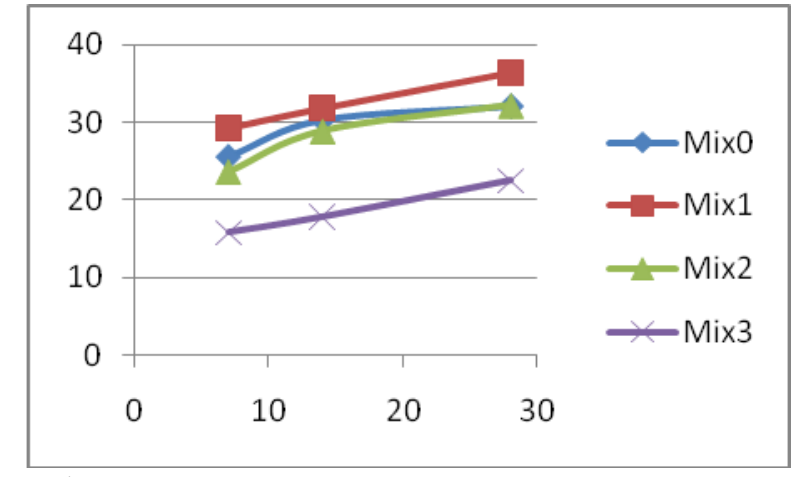

الشكل 1: نتائج فحص مقاومة الإنضغاط لخلطات الطريقة الأولى.

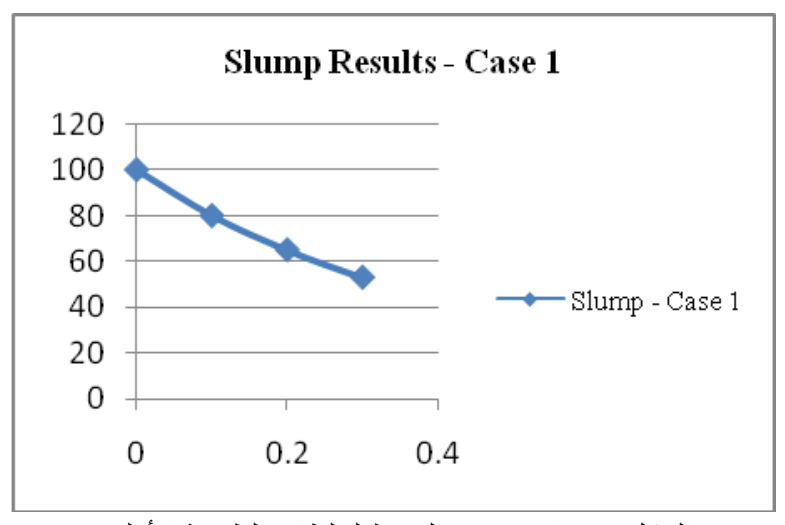

الثكل 2: نتائج فحص الهبوط لخلطات الطريقة الأولى. 


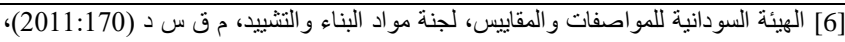

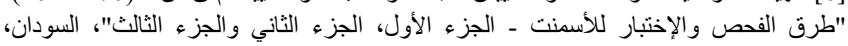

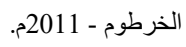

[7] ACI Committee Report 226. IR - 87, "Ground Granulated - Blast Furnace Slag as a Cementitious Constituent in Concrete", ACI Materials Journal, July - August 1987.

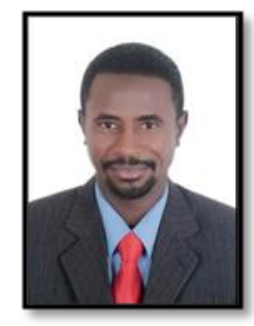

\section{Dr. Ali Hussein Mohamed Ali}

University of Bahri, College of Engineering \& Architecture. Department of Civil Engineering. Date of birth: 1971 - Elobeid (N. K. state) Ph.D in Structural Engineering - Karary University [2011]. M.Sc in Structural Engineering - SUST [2001]. B.Sc in Structural Engineering - SUST [1996]. Major field of study: Civil Engineering [Structures].

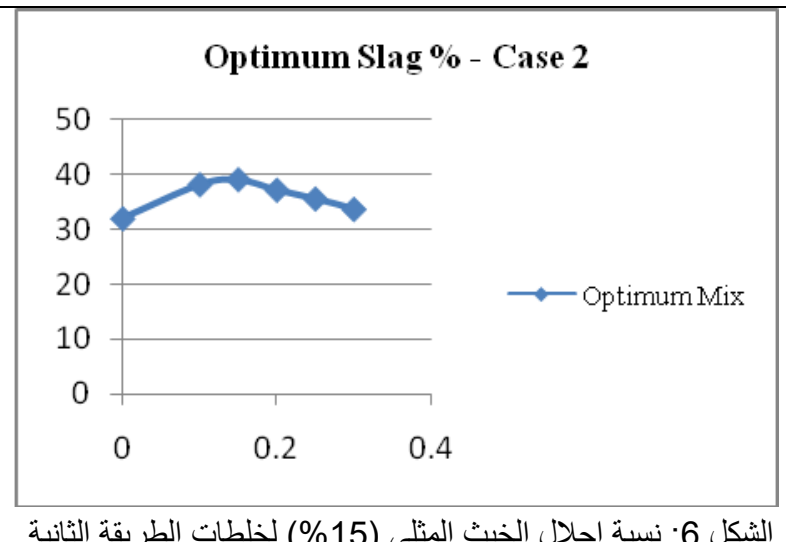

الثكل 6: نسبة إحلال الخبث المثلى (15\%) لخطات الطريقة الثانية.

3- إنخفاض قابلية التشغيل لجميع الخلطات الخرسانية مع إحلال

الخبث كجزء من الأسمنت، حيث أظهرت نتائج فحص الهجوط أن إحلال الخبث يعطى قابلية تشغيل أقل من الخلطة المرجعية. 4- إستبدال جزء من الأسمنت بالخبث وذلك من أجل تقليل التكلفة وتقليل معدل إنبعاث الحرارة وخاصة في الخلطات ذات المحتوى العالي من الأسمنت.

5- ملائمة إستخدام الخبث كمكون فى إنتاج الخرسانة مع إضافة الملدنات المتفوقة وهذا يتفق مع ما توصلت إليه الدراسة.

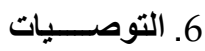

من خلال نتائج الدراسـة والإطلاع على الدر اسـات و البحوث القليلة عن الخبث المحلى نستخلص التوصيات الآتية:

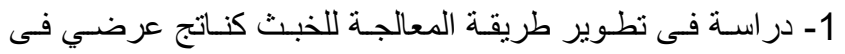

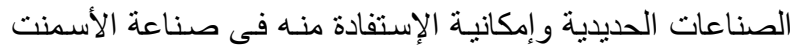
المقاوم وخاصة إنه يحتوي على نسبة عالية من أكاسيد الحديد.

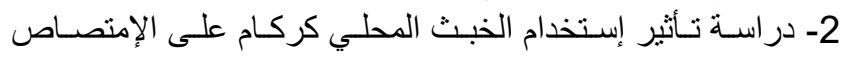
ومسامية الخرسانة عالية الأداء.

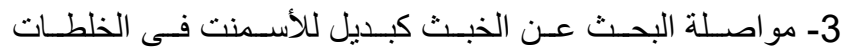

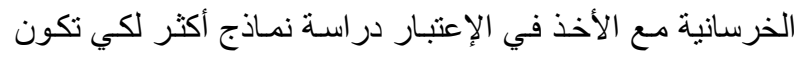
الدر اسة ذات أساس علمي و اضح الاضح.

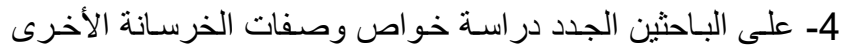

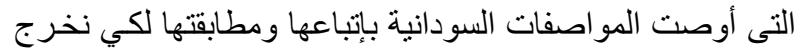
بإستنتاجات موثوقة.

\section{المر اجع والمصادر}

[1] ACI Committee Report 226. IR - 87, "Ground Granulated Blast Furnace Slag as a Cementitious Constituent in Concrete", ACI Materials Journal, July - August, PP 327-342, (1987).

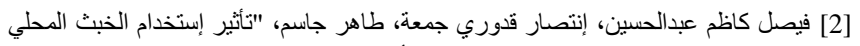

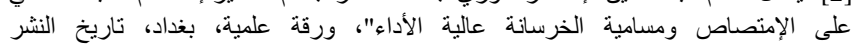
2009/04/20

[3] Lea, F.M., "The Chemistry of Cement And Concrete", Third edition Edward Arnohd, London ,PP ,454 - 590, 557 - 587, (1970).

[4] Mather B., "Laboratory test of Portland Blast Furnace Slag Cement", ACI Journal, Proc.V.45 . No. 3 Sept. PP. 05 - 232, (1957).

[5] Lee. A. R.. "Blast Furnace and Steel Slag, Production, Properties And Uses", Edward Arnold Publish Ltd., London, (1974). 